\title{
Global Existence and Convergence Rates for the 3-D Compressible Navier-Stokes Equations Without Heat Conductivity
}

\author{
Renjun Duan* and Hongfang Ma \\ Department of Mathematics, City University of Hong Kong, \\ 83 Tat Chee Avenue, Kowloon, Hong Kong, P.R. China
}

2007-06-04

\begin{abstract}
We study the global existence and convergence rates of solutions to the three-dimensional compressible Navier-Stokes equations without heat conductivity, which is a hyperbolic-parabolic system. The pressure and velocity are dissipative because of the viscosity, whereas the entropy is non-dissipative due to the absence of heat conductivity. The global solutions are obtained by combining the local existence and a priori estimates if $H^{3}$-norm of the initial perturbation around a constant state is small enough and its $L^{1}$-norm is bounded. A priori decay-in-time estimates on the pressure and velocity are used to get the uniform bound of entropy. Moreover, the optimal convergence rates are also obtained.
\end{abstract}

Keywords: Navier-Stokes equations; a priori estimate; convergence rates.

AMS Subject Classification: 35Q30, 65M12, 93D20

\section{Introduction}

In the whole space $\mathbb{R}^{3}$, the compressible Navier-Stokes equations for the density $\rho$, the velocity $u=\left(u_{1}, u_{2}, u_{3}\right)$ and the temperature $\theta$, which determine the motion of gases, are written as

$$
\left\{\begin{array}{l}
\rho_{t}+\nabla \cdot(\rho u)=0, \\
\rho\left[u_{t}+(u \cdot \nabla) u\right]+\nabla p(\rho, \theta)=\mu \Delta u+\left(\mu+\mu^{\prime}\right) \nabla(\nabla \cdot u), \\
\rho c_{V}\left[\theta_{t}+(u \cdot \nabla) \theta\right]+\theta p_{\theta}(\rho, \theta) \nabla \cdot u=\kappa \Delta \theta+\Psi(u),
\end{array}\right.
$$

*Email: mathrjduan@hotmail.com 
where $p=p(\rho, \theta)$ is the pressure, $\mu$ and $\mu^{\prime}$ are the viscosity coefficients, $\kappa$ is the coefficient of heat conduction, $c_{V}$ is the specific heat at constant volume, and $\Psi=$ $\Psi(u)$ is the classical dissipation function:

$$
\Psi(u)=\frac{\mu}{2} \sum_{i, j=1}^{3}\left(\partial_{i} u_{j}+\partial_{j} u_{i}\right)^{2}+\mu^{\prime} \sum_{j=1}^{3}\left(\partial_{j} u_{j}\right)^{2} .
$$

In this paper, we study the case when the coefficient of heat conduction $\kappa=0$, the viscosity coefficients $\mu>0$ and $\mu^{\prime}$ are constants with $\mu^{\prime}+\frac{2}{3} \mu \geq 0$, the specific heat $c_{V}>0$ is a constant, and the gas is ideal and polytropic, i.e.

$$
p=\rho \theta, \quad e=c_{V} \theta,
$$

where $e$ is the internal energy.

It is well-known that all thermodynamics variables $\rho, \theta, e, p$ as well as the entropy $s$ can be denoted by functions of any two of them. We take the two variables to be $p$ and $s$. Then the equation of state for the gas is then given by

$$
\rho=a p^{\frac{c_{V}}{c_{V}+1}} \exp \left(-\frac{s}{c_{V}+1}\right)
$$

where $a>0$ is a constant. Under the aforementioned assumptions, the system (1.1) in terms of the variables $p, u$ and $s$ reads

$$
\left\{\begin{array}{l}
p_{t}+\frac{1+c_{V}}{c_{V}} p \nabla \cdot u+u \cdot \nabla p=\frac{\Psi(u)}{c_{V}}, \\
u_{t}+(u \cdot \nabla) u+\frac{\nabla p}{\rho}=\frac{\mu}{\rho} \Delta u+\frac{\mu+\mu^{\prime}}{\rho} \nabla(\nabla \cdot u), \\
s_{t}+(u \cdot \nabla) s=\frac{\Psi(u)}{p}
\end{array}\right.
$$

where $\rho=\rho(p, s)$ is defined by (1.2). Notice that (1.3) is a hyperbolic-parabolic system, where the dissipation comes from viscosity. We consider the initial value problem to (1.3) in the whole space $\mathbb{R}^{3}$ with the initial data

$$
(p, u, s)(0, x)=\left(p_{0}, u_{0}, s_{0}\right)(x) \rightarrow\left(p_{\infty}, 0, s_{\infty}\right) \text { as }|x| \rightarrow \infty,
$$

where $p_{\infty}>0$ and $s_{\infty}$ are given constants.

The main purpose of the paper is to prove the following theorem concerning the global existence and the convergence rates of solutions to the initial value problem (1.3) and (1.4) when the initial data is a small perturbation of a constant state.

Theorem 1.1. Let the initial data $\left(p_{0}, u_{0}, s_{0}\right)$ be such that $\left\|\left(p_{0}-p_{\infty}, u_{0}, s_{0}-s_{\infty}\right)\right\|_{3}$ is sufficiently small and $\left\|\left(p_{0}-p_{\infty}, u_{0}\right)\right\|_{L^{1}}$ is bounded. Then the initial value problem (1.3)-(1.4) admits a unique solution $(p, u, s)$ globally in time with $p>0$, satisfying

$$
\begin{array}{r}
p-p_{\infty}, s-s_{\infty} \in C^{0}\left(0, \infty ; H^{3}\left(\mathbb{R}^{3}\right)\right) \cap C^{1}\left(0, \infty ; H^{2}\left(\mathbb{R}^{3}\right)\right), \\
u \in C^{0}\left(0, \infty ; H^{3}\left(\mathbb{R}^{3}\right)\right) \cap C^{1}\left(0, \infty ; H^{1}\left(\mathbb{R}^{3}\right)\right) .
\end{array}
$$


Moreover, there exists a constant $C_{0}$ such that for any $t \geq 0$,

$$
\begin{gathered}
\left\|\left(p-p_{\infty}, u\right)(t)\right\|_{3}^{2}+\int_{0}^{t}\left(\|\nabla p(\tau)\|_{2}^{2}+\|\nabla u(\tau)\|_{3}^{2}\right) d \tau \leq C_{0}\left\|\left(p_{0}-p_{\infty}, u_{0}\right)\right\|_{3}^{2}, \\
\left\|\left(s-s_{\infty}\right)(t)\right\|_{3} \leq C_{0}\left\|\left(p_{0}-p_{\infty}, u_{0}, s-s_{\infty}\right)\right\|_{3} \exp \left(C_{0}\left\|\left(p_{0}-p_{\infty}, u_{0}\right)\right\|_{L^{1} \cap H^{3}}\right) .
\end{gathered}
$$

Finally, there is a constant $C_{0}^{\prime}$ such that for any $t \geq 0$, the solution $(p, u, s)$ has the decay properties

$$
\begin{aligned}
\left\|\left(p-p_{\infty}, u\right)(t)\right\|_{L^{q}} & \leq C_{0}^{\prime}(1+t)^{-\frac{3}{2}\left(1-\frac{1}{q}\right)}, \quad 2 \leq q \leq 6 \\
\left\|\nabla\left(p-p_{\infty}, u\right)(t)\right\|_{2} & \leq C_{0}^{\prime}(1+t)^{-\frac{5}{4}} \\
\left\|\left(p-p_{\infty}, u\right)(t)\right\|_{L^{\infty}} & \leq C_{0}^{\prime}(1+t)^{-\frac{5}{4}} \\
\left\|\partial_{t}(p, u, s)(t)\right\| & \leq C_{0}^{\prime}(1+t)^{-\frac{5}{4}}
\end{aligned}
$$

Remark 1.1. The boundedness of $\left\|\left(p_{0}-p_{\infty}, u_{0}\right)\right\|_{L^{1}}$ is used in the proof of the global existence. This is different from the previous work [15] for the case of $\kappa>0$, where only $\mathrm{H}^{3}$-norm of the perturbation is supposed for the global existence. In addition, due to lack of heat conductivity, the entropy $s$ is non-dissipative and thus has no decay-in-time property. However, all time derivatives $\partial_{t}(p, u, s)$ in $L^{2}$-norm decay in time.

The study of this paper is motivated by Liu-Zeng [13], where the authors considered the one-dimensional compressible Navier-Stokes equaions without heat conductivity in the Lagrangian coordinates

$$
\left\{\begin{array}{l}
\partial_{t} \rho+\rho^{2} \partial_{x} u=0 \\
\partial_{t} u+\partial_{x} p=\partial_{x}\left(\mu \rho \partial_{x} u\right) \\
\partial_{t} E+\partial_{x}(p u)=\partial_{x}\left(\mu \rho u \partial_{x} u\right)
\end{array}\right.
$$

which in terms of $(p, u, s)$, also read

$$
\left\{\begin{array}{l}
\partial_{t} p+c^{2} \partial_{x} u=\mu \rho p_{e}\left(\partial_{x} u\right)^{2} \\
\partial_{t} u+\partial_{x} p=\partial_{x}\left(\mu \rho \partial_{x} u\right) \\
\partial_{t} s=\frac{\mu}{\theta} \rho\left(\partial_{x} u\right)^{2}
\end{array}\right.
$$

In [13], the elaborate pointwise estimates and large-time behavior of solutions to (1.5) were obtained by studying the Green's function and the nonlinear interaction of waves. We also mention the work of Kawashima [8], where the global existence of (1.5) was also announced. However, in this paper we are concerned with the global existence and convergence rates of solutions for the case of three spatial dimensions. It is observed that in the Euler's coordinates, the dissipative variables $p$ and $u$ satisfy $(1.3)_{1}-(1.3)_{2}$ whose linear parts possess the same structure as ones of the isentropic viscous compressible Navier-Stokes equations, and the non-dissipative variable $s$ satisfies the transport equation $(1.3)_{3}$ with the nonlinear source term. Thus in order 
to obtain the global existence of solutions to (1.3), the direct energy method as in [16] can apply to the first two equations of (1.3) to obtain the uniform bound of $(p, u)$ under a priori assumption that $\|(p, u, s)(t)\|_{3}$ is sufficiently small; see Lemmas 4.1 and 4.2 in Section 4. Furthermore, the uniform bound of $s$ will be obtained by making a priori decay-in-time estimates on $(p, u)$, which is based on the decay property of the linearized equations together with energy estimates of higher order; see Section 5. This kind of method can be found in the recent works in $[4,5]$ about the study of optimal convergence rates for the compressible Navier-Stokes equations with a potential force. Different from $[4,5]$, in this paper the additional condition on the $L^{1}$-norm boundedness of the initial perturbation plays a role not only in the proof of the convergence rates but also in the proof of the global existence. Roughly, the reason why we need it is the appearance of the transport term $u \cdot \nabla s$ in $(1.3)_{3}$.

There are a lot of works on the existence, stability and convergence rates of solutions to the compressible Navier-Stokes equations. Here we only mention some of them related to our study for the case of the whole space. When $\kappa>0$, the problem was studied by Matsumura and Nishida [15, 16], Ponce [17], Deckelnick $[2,3]$, Hoff-Zumbrun [6, 7], Liu-Wang [12]. In the presence of the potential force, the almost optimal convergence rates were considered by [18] and the optimal ones by $[4,5]$. When $\kappa=0$, the one-dimensional system in the Lagrangian coordinates was studied by $[13,14,20]$ and some references therein; see also [19] about a relaxation model for a gas in thermal non-equilibrium.

The rest of the paper is organized as follows. We will reformulate the problem in Section 2. In Section 3, we list some elementary facts on the decay-in-time estimates on $(p, u)$ for the linearized equations and some useful inequalities. The proof of Theorem 1.1 is given in Sections 4 and 5.

Notations. Throughout this paper, the norms in the Sobolev Spaces $H^{m}\left(\mathbb{R}^{3}\right)$ and $W^{m, q}\left(\mathbb{R}^{3}\right)$ are denoted respectively by $\|\cdot\|_{m}$ and $\|\cdot\|_{m, q}$ for $m \geq 0$ and $q \geq 1$. In particular, for $m=0$, we will simply use $\|\cdot\|$ and $\|\cdot\|_{L^{q}} \cdot\langle\cdot, \cdot\rangle$ denotes the inner-product in $L^{2}\left(\mathbb{R}^{3}\right)$. Moreover, $C$ denotes a general constant which may vary in different estimates. Finally,

$$
\nabla=\left(\partial_{1}, \partial_{2}, \partial_{3}\right), \quad \partial_{i}=\partial_{x_{i}}, i=1,2,3,
$$

and for any integer $l \geq 0, \nabla^{l} f$ denotes all derivatives of order $l$ of the function $f$. And for multi-indices $\alpha$ and $\beta$

$$
\alpha=\left(\alpha_{1}, \alpha_{2}, \alpha_{3}\right), \quad \beta=\left(\beta_{1}, \beta_{2}, \beta_{3}\right),
$$

we use

$$
\partial_{x}^{\alpha}=\partial_{x_{1}}^{\alpha_{1}} \partial_{x_{2}}^{\alpha_{2}} \partial_{x_{3}}^{\alpha_{3}}, \quad|\alpha|=\sum_{i=1}^{3} \alpha_{i},
$$

and $C_{\alpha}^{\beta}=\frac{\alpha !}{\beta !(\alpha-\beta) !}$ when $\beta \leq \alpha$. 


\section{Reformulated system}

In this section, we will first reformulate the problem as follows. Set

$$
\lambda=\sqrt{\frac{c_{V}}{\left(1+c_{V}\right) \rho_{\infty} p_{\infty}}}, \lambda_{1}=\sqrt{\frac{\left(1+c_{V}\right) p_{\infty}}{c_{V} \rho_{\infty}}}, \mu_{1}=\frac{\mu}{\rho_{\infty}}, \mu_{2}=\frac{\mu+\mu^{\prime}}{\rho_{\infty}},
$$

where $\rho_{\infty}=\rho\left(p_{\infty}, s_{\infty}\right)$. Taking change of variables by $(p, u, s) \rightarrow\left(p+p_{\infty}, \lambda u, s+s_{\infty}\right)$, the initial value problem (1.3)-(1.4) is reformulated as

$$
\left\{\begin{array}{l}
p_{t}+\lambda_{1} \nabla \cdot u=g_{1} \\
u_{t}+\lambda_{1} \nabla p-\mu_{1} \Delta u-\mu_{2} \nabla \nabla \cdot u=g_{2}, \\
s_{t}+\lambda(u \cdot \nabla) s=g_{3} \\
(p, u, s)(0, x)=\left(p_{0}, u_{0}, s_{0}\right)(x) \rightarrow(0,0,0) \text { as }|x| \rightarrow \infty
\end{array}\right.
$$

where

$$
\begin{aligned}
g_{1}(p, u, s) \equiv & -\frac{\left(1+c_{V}\right) \lambda}{c_{V}} p \nabla \cdot u-\lambda u \cdot \nabla p+\frac{\Psi(\lambda u)}{c_{V}} \\
g_{2}(p, u, s) \equiv & -\lambda u \cdot \nabla u-\frac{1}{\lambda}\left(\frac{1}{\rho}-\frac{1}{\rho_{\infty}}\right) \nabla p+\mu\left(\frac{1}{\rho}-\frac{1}{\rho_{\infty}}\right) \Delta u \\
& +\left(\mu+\mu^{\prime}\right)\left(\frac{1}{\rho}-\frac{1}{\rho_{\infty}}\right) \nabla \nabla \cdot u, \\
g_{3}(p, u, s) \equiv & \frac{\Psi(\lambda u)}{p+p_{\infty}} .
\end{aligned}
$$

Here and in the sequel, for the notational simplicity, we still denote the reformulated variables by $(p, u, s)$.

Let us define the solution space and the solution norm of the initial value problem (2.1) by

$$
\begin{aligned}
X(0, T)= & \left\{(p, u, s) ; p, s \in C^{0}\left(0, T ; H^{3}\left(\mathbb{R}^{3}\right)\right) \cap C^{1}\left(0, T ; H^{2}\left(\mathbb{R}^{3}\right)\right),\right. \\
& u \in C^{0}\left(0, T ; H^{3}\left(\mathbb{R}^{3}\right)\right) \cap C^{1}\left(0, T ; H^{1}\left(\mathbb{R}^{3}\right)\right), \\
& \left.\nabla p \in L^{2}\left(0, T ; H^{2}\left(\mathbb{R}^{3}\right)\right), \nabla u \in L^{2}\left(0, T ; H^{3}\left(\mathbb{R}^{3}\right)\right)\right\},
\end{aligned}
$$

and

$$
N(0, T)^{2}=\sup _{0 \leq t \leq T}\|(p, u, s)(t)\|_{3}^{2}+\int_{0}^{T}\left(\|\nabla p(t)\|_{2}^{2}+\|\nabla u(t)\|_{3}^{2}\right) d t,
$$

for any $0 \leq T \leq \infty$. As usual, the global existence of solution to (2.1) will be obtained by combining the local existence result together with a priori estimates.

Proposition 2.1 (Local existence). Let $\left(p_{0}, u_{0}, s_{0}\right) \in H^{3}\left(\mathbb{R}^{3}\right)$ be such that

$$
\inf _{x \in \mathbb{R}^{3}}\left\{p_{0}(x)+p_{\infty}\right\}>0
$$


Then there exists a positive constant $T_{0}$ depending on $N(0,0)$ such that the initial value problem $(2.1)$ has a unique solution $(p, u, s) \in X\left(0, T_{0}\right)$ which satisfies $N\left(0, T_{0}\right) \leq 2 N(0,0)$ and

$$
\inf _{x \in \mathbb{R}^{3}, 0 \leq t \leq T_{0}}\left\{p(t, x)+p_{\infty}\right\}>0 .
$$

Proposition 2.2 (A priori estimate). Let $\left(p_{0}, u_{0}, s_{0}\right) \in H^{3}\left(\mathbb{R}^{3}\right)$ and $\left(p_{0}, u_{0}\right) \in$ $L^{1}\left(\mathbb{R}^{3}\right)$. Suppose that the initial value problem $(2.1)$ has a solution $(p, u, s) \in$ $X(0, T)$, where $T$ is a positive constant. Then there exist a small constant $\epsilon>0$ and a constant $C_{1}$, which are independent of $T$, such that if

$$
\sup _{0 \leq t \leq T}\|(p, u, s)(t)\|_{3} \leq \epsilon,
$$

then for any $t \in[0, T]$, it holds that

$$
\begin{gathered}
\|(p, u)(t)\|_{3}^{2}+\int_{0}^{t}\left(\|\nabla p(\tau)\|_{2}^{2}+\|\nabla u(\tau)\|_{3}^{2}\right) d \tau \leq C_{1}\left\|\left(p_{0}, u_{0}\right)\right\|_{3}^{2}, \\
\|s(t)\|_{3} \leq C_{1}\left\|\left(p_{0}, u_{0}, s_{0}\right)\right\|_{3} \exp \left(C_{1} K_{0}\right),
\end{gathered}
$$

where

$$
K_{0}=\left\|\left(p_{0}, u_{0}\right)\right\|_{L^{1} \cap H^{3}} .
$$

Furthermore, there is a constant $C_{1}^{\prime}$ such that for any $t \in[0, T]$, the solution $(p, u, s)$ has the decay properties

$$
\begin{aligned}
\|(p, u)(t)\|_{L^{q}} & \leq C_{1}^{\prime} K_{0}(1+t)^{-\frac{3}{2}\left(1-\frac{1}{q}\right)}, \quad 2 \leq q \leq 6, \\
\|\nabla(p, u)(t)\|_{2} & \leq C_{1}^{\prime} K_{0}(1+t)^{-\frac{5}{4}} \\
\|(p, u)(t)\|_{L^{\infty}} & \leq C_{1}^{\prime} K_{0}(1+t)^{-\frac{5}{4}} \\
\left\|\partial_{t}(p, u, s)(t)\right\| & \leq C_{1}^{\prime} K_{0}(1+t)^{-\frac{5}{4}}
\end{aligned}
$$

Theorem 1.1 follows from Proposition 2.1 and Proposition 2.2 by the standard continuity argument. The proof of Proposition 2.1 is standard and thus omitted; see $[8,9]$ for the study of the local existence on the general hyperbolic-parabolic systems. Proposition 2.2 will be proved in Section 4 and Section 5 .

\section{$3 \quad$ Elementary}

The linearized equations corresponding to $(2.1)_{1}-(2.1)_{3}$ read

$$
\left\{\begin{array}{l}
p_{t}+\lambda_{1} \nabla \cdot u=0, \\
u_{t}+\lambda_{1} \nabla p-\mu_{1} \Delta u-\mu_{2} \nabla \nabla \cdot u=0, \\
s_{t}=0 .
\end{array}\right.
$$

Thus, at the level of the linearization, $s$ is decoupled with $(p, u)$. If we set

$$
U(t)=(p(t), u(t)),
$$


then the solution to $(3.1)_{1}-(3.1)_{2}$ can be written as

$$
U(t)=e^{-t \mathbb{A}} U(0),
$$

where $\mathbb{A}$ is a matrix-valued differential operator given by

$$
\mathbb{A}=\left(\begin{array}{cc}
0 & \lambda_{1} \operatorname{div} \\
\lambda_{1} \nabla & -\mu_{1} \Delta-\mu_{2} \nabla \operatorname{div}
\end{array}\right) .
$$

The solution semigroup $e^{-\mathbb{A} t}$ has the following property on the decay in time, cf. $[10,11]$.

Lemma 3.1. Let $k \geq 0$ be an integer and $1 \leq l \leq 2$. Then for any $t \geq 0$, it holds that

$$
\left\|\nabla^{k} e^{-t \mathbb{A}} U(0)\right\| \leq C(1+t)^{-\sigma(l, 2 ; k)}\|U(0)\|_{L^{l} \cap H^{k}},
$$

where the decay rate is measured by

$$
\sigma(l, 2 ; k)=\frac{3}{2}\left(\frac{1}{l}-\frac{1}{2}\right)+\frac{k}{2} .
$$

For later use we list some Sobolev inequalities as follows, cf. [1, 2].

Lemma 3.2. Let $f \in H^{2}\left(\mathbb{R}^{3}\right)$. Then

(i) $\|f\|_{L^{\infty}} \leq C\|\nabla f\|^{\frac{1}{2}}\|\nabla f\|_{1}^{\frac{1}{2}} \leq C\|\nabla f\|_{1}$;

(ii) $\|f\|_{L^{6}} \leq C\|\nabla f\|$;

(iii) $\|f\|_{L^{q}} \leq C\|f\|_{1}, 2 \leq q \leq 6$.

Finally, the following elementary inequality also will be used, cf. [5].

Lemma 3.3. If $r_{1}>1$ and $r_{2} \in\left[0, r_{1}\right]$, then it holds that

$$
\int_{0}^{t}(1+t-\tau)^{-r_{1}}(1+\tau)^{-r_{2}} d \tau \leq C\left(r_{1}, r_{2}\right)(1+t)^{-r_{2}} .
$$

\section{A priori estimates}

Throughout this section and next section, we suppose that all the conditions of Proposition 2.2 hold. The initial value problem $(2.1)$ has a solution $(p, u, s)$ in the space $X(0, T)$ with some $T \in(0,+\infty]$. Furthermore we make a priori assumption

$$
\sup _{0 \leq t \leq T}\|(p, u, s)(t)\|_{3} \leq \epsilon,
$$

where $\epsilon>0$ is sufficiently small. For simplicity, in this section we omit the variable $t$ of all functions in the proof of different lemmas.

In what follows, a series of lemmas on the energy estimates are given. Firstly, the energy estimate of lower order for $(p, u)$ is obtained in the following lemma. 
Lemma 4.1. There exists a constant $D_{1}>0$ suitably large which is independent of $\epsilon$ such that

$$
\frac{d}{d t}\left(D_{1}\|(p, u)(t)\|^{2}+\langle\nabla p, u\rangle(t)\right)+C\|\nabla(p, u)(t)\|^{2} \leq C\left\|\nabla^{2} u(t)\right\|^{2},
$$

for any $0 \leq t \leq T$.

Proof. Multiply $(2.1)_{1}-(2.1)_{2}$ by $p, u$ respectively and then integrating them over $\mathbb{R}^{3}$, we have

$$
\frac{1}{2} \frac{d}{d t}\|(p, u)\|^{2}+\mu_{1}\|\nabla u\|^{2}+\mu_{2}\|\nabla \cdot u\|^{2}=\left\langle p, g_{1}\right\rangle+\left\langle u, g_{2}\right\rangle .
$$

The two terms on the right hand side of the above equation can be estimated as follows.

Firstly, for the first term, it holds that

$$
\begin{aligned}
\left\langle p, g_{1}\right\rangle & =-\frac{\left(1+c_{V}\right) \lambda}{c_{V}}\langle p, p \nabla \cdot u\rangle-\lambda\langle p, u \cdot \nabla p\rangle+\frac{1}{c_{V}}\langle p, \Psi(\lambda u)\rangle \\
& =\left\{-\frac{\left(1+c_{V}\right) \lambda}{c_{V}}+\frac{\lambda}{2}\right\}\left\langle p^{2}, \nabla \cdot u\right\rangle+\frac{\lambda^{2}}{c_{V}}\langle p, \Psi(u)\rangle .
\end{aligned}
$$

It follows from Lemma 3.2, Hölder inequality and (4.1) that

$$
\begin{aligned}
\left|\left\langle p^{2}, \nabla \cdot u\right\rangle\right| & \leq\|p\|_{L^{3}}\|p\|_{L^{6}}\|\nabla u\| \leq C\|p\|_{1}\|\nabla p\|\|\nabla u\| \\
& \leq C \epsilon\left(\|\nabla p\|^{2}+\|\nabla u\|^{2}\right),
\end{aligned}
$$

and

$$
|\langle p, \Psi(u)\rangle| \leq C\|p\|_{L^{\infty}}\|\nabla u\|^{2} \leq C\|\nabla p\|_{1}\|\nabla u\|^{2} \leq C \epsilon\|\nabla u\|^{2},
$$

which together with (4.4) implies that the first term is bounded by

$$
\left|\left\langle p, g_{1}\right\rangle\right| \leq C \epsilon\left(\|\nabla p\|^{2}+\|\nabla u\|^{2}\right) .
$$

For the second term, we have

$$
\begin{aligned}
\left|\left\langle u, g_{2}\right\rangle\right| \leq & C\left\{|\langle u, u \cdot \nabla u\rangle|+\left|\left\langle u,\left(\frac{1}{\rho}-\frac{1}{\rho_{\infty}}\right) \nabla p\right\rangle\right|\right. \\
& \left.+\left|\left\langle u,\left(\frac{1}{\rho}-\frac{1}{\rho_{\infty}}\right) \Delta u\right\rangle\right|+\left|\left\langle u,\left(\frac{1}{\rho}-\frac{1}{\rho_{\infty}}\right) \nabla \nabla \cdot u\right\rangle\right|\right\} .
\end{aligned}
$$

Similar to the proof of (4.5), it follows from Lemma 3.2, Hölder inequality and (4.1) that

$$
\begin{aligned}
|\langle u, u \cdot \nabla u\rangle| & \leq\|u\|_{L^{3}}\|u\|_{L^{6}}\|\nabla u\| \leq C\|u\|_{1}\|\nabla u\|^{2} \leq C \epsilon\|\nabla u\|^{2}, \\
\left|\left\langle u,\left(\frac{1}{\rho}-\frac{1}{\rho_{\infty}}\right) \nabla p\right\rangle\right| & \leq\|u\|_{L^{6}}\left\|\frac{1}{\rho}-\frac{1}{\rho_{\infty}}\right\|_{L^{3}}\|\nabla p\| \\
& \leq C\|\nabla u\|\|(p, s)\|\left\|_{1}\right\| \nabla p \| \\
& \leq C \epsilon\left(\|\nabla p\|^{2}+\|\nabla u\|^{2}\right), \\
\left|\left\langle u,\left(\frac{1}{\rho}-\frac{1}{\rho_{\infty}}\right) \Delta u\right\rangle\right| & =\left|\left\langle u, \nabla\left(\frac{1}{\rho}-\frac{1}{\rho_{\infty}}\right) \cdot \nabla u\right\rangle+\left\langle\nabla u,\left(\frac{1}{\rho}-\frac{1}{\rho_{\infty}}\right) \nabla u\right\rangle\right| \\
& \leq C\left\{\|\nabla(p, s)\|_{1}\|\nabla u\|^{2}+\|(p, s)\|_{L^{\infty}}\|\nabla u\|^{2}\right\} \\
& \leq C\|\nabla(p, s)\|_{1}\|\nabla u\|^{2} \\
& \leq C \epsilon\|\nabla u\|^{2},
\end{aligned}
$$


and similarly,

$$
\left|\left\langle u,\left(\frac{1}{\rho}-\frac{1}{\rho_{\infty}}\right) \nabla \nabla \cdot u\right\rangle\right| \leq C \epsilon\|\nabla u\|^{2},
$$

where by (1.2) and (4.1), we have used the fact

$$
\frac{1}{\rho}-\frac{1}{\rho_{\infty}} \sim O(1)(p+s) .
$$

Substituting (4.7) and (4.8) into (4.6) gives that the second term is bounded by

$$
\left|\left\langle u, g_{2}\right\rangle\right| \leq C \epsilon\left(\|\nabla p\|^{2}+\|\nabla u\|^{2}\right) .
$$

Hence combining (4.3), (4.5) and (4.9) yields

$$
\frac{d}{d t}\|(p, u)\|^{2}+C\|\nabla u\|^{2} \leq C \epsilon\|\nabla p\|^{2},
$$

since $\epsilon>0$ is sufficiently small.

Next we shall estimate $\|\nabla p\|^{2}$. From $(2.1)_{2}$, one has

$$
\lambda_{1}\|\nabla p\|^{2}=\left\langle-u_{t}, \nabla p\right\rangle+\mu_{1}\langle\Delta u, \nabla p\rangle+\mu_{2}\langle\nabla \nabla \cdot u, \nabla p\rangle+\left\langle g_{2}, \nabla p\right\rangle,
$$

where from $(2.1)_{1}$, the first term on the right hand side can be written as

$$
\begin{aligned}
\left\langle-u_{t}, \nabla p\right\rangle & =-\frac{d}{d t}\langle\nabla p, u\rangle+\left\langle\nabla p_{t}, u\right\rangle \\
& =-\frac{d}{d t}\langle\nabla p, u\rangle-\left\langle p_{t}, \nabla \cdot u\right\rangle \\
& =-\frac{d}{d t}\langle\nabla p, u\rangle+\left\langle\lambda_{1} \nabla \cdot u-g_{1}, \nabla \cdot u\right\rangle .
\end{aligned}
$$

It follows from the definition of $g_{1}$ that

$$
\begin{aligned}
\left|\left\langle-g_{1}, \nabla \cdot u\right\rangle\right| & \leq C|\langle p \nabla \cdot u, \nabla \cdot u\rangle|+|\langle u \cdot \nabla p, \nabla \cdot u\rangle|+|\langle\Psi(\lambda u), \nabla \cdot u\rangle| \\
& \leq C\left\{\|\nabla p\|_{1}\|\nabla \cdot u\|^{2}+\|\nabla p\|_{1}\|\nabla u\|\|\nabla \cdot u\|+\|\nabla u\|^{2}\|\nabla \nabla \cdot u\|_{1}\right\} \\
& \leq C \epsilon\|\nabla u\|^{2} .
\end{aligned}
$$

Then (4.11)-(4.12) give

$$
\begin{aligned}
& \lambda_{1}\|\nabla p\|^{2}+\frac{d}{d t}\langle\nabla p, u\rangle \\
= & \mu_{1}\langle\Delta u, \nabla p\rangle+\mu_{2}\langle\nabla \nabla \cdot u, \nabla p\rangle+\left\langle\lambda_{1} \nabla \cdot u-g_{1}, \nabla \cdot u\right\rangle+\left\langle g_{2}, \nabla p\right\rangle \\
\leq & C\left\|\nabla^{2} u\right\|^{2}+\frac{\lambda_{1}}{4}\|\nabla p\|^{2}+\lambda_{1}\|\nabla \cdot u\|^{2}+C \epsilon\|\nabla u\|^{2}+\left|\left\langle g_{2}, \nabla p\right\rangle\right| .
\end{aligned}
$$

Similar to the estimate on $\left\langle g_{2}, u\right\rangle$, we have

$$
\left|\left\langle g_{2}, \nabla p\right\rangle\right| \leq C \epsilon\left(\|\nabla p\|^{2}+\|\nabla u\|^{2}\right)+C \epsilon\left\|\nabla^{2} u\right\|^{2} .
$$

Since $\epsilon>0$ is small enough, putting (4.14) into (4.13) gives

$$
\frac{\lambda_{1}}{2}\|\nabla p\|^{2}+\frac{d}{d t}\langle\nabla p, u\rangle \leq C \epsilon\|\nabla u\|^{2}+C\left\|\nabla^{2} u\right\|^{2} .
$$

Finally, multiplying (4.10) by $D_{1}$ suitably large and adding it to (4.15), one has (4.2) since $\epsilon>0$ is sufficiently small. This completes the proof of the lemma. 
Next, in the following lemma we give the energy estimate of the higher order for $(p, u)$.

Lemma 4.2. There exists a constant $D_{2}>0$ sufficiently large which is independent of $\epsilon$ such that

$$
\begin{aligned}
& \frac{d}{d t}\left(D_{2}\|\nabla(p, u)(t)\|_{2}^{2}+\sum_{1 \leq|\alpha| \leq 2}\left\langle\nabla \partial_{x}^{\alpha} p, \partial_{x}^{\alpha} u\right\rangle(t)\right) \\
& +C\left(\left\|\nabla^{2} p(t)\right\|_{1}^{2}+\left\|\nabla^{2} u(t)\right\|_{2}^{2}\right) \leq C \epsilon\|\nabla(p, u)(t)\|^{2},
\end{aligned}
$$

for any $0 \leq t \leq T$.

Proof. For each multi-index $\alpha$ with $1 \leq|\alpha| \leq 3$, by applying $\partial_{x}^{\alpha}$ to $(2.1)_{1}-(2.1)_{2}$, multiplying them by $\partial_{x}^{\alpha} p, \partial_{x}^{\alpha} u$ respectively and then integrating them over $\mathbb{R}^{3}$, we have

$$
\begin{aligned}
& \frac{1}{2} \frac{d}{d t}\left\|\partial_{x}^{\alpha}(p, u)\right\|^{2}+\mu_{1}\left\|\nabla \partial_{x}^{\alpha} u\right\|^{2}+\mu_{2}\left\|\nabla \cdot \partial_{x}^{\alpha} u\right\|^{2} \\
& =\left\langle\partial_{x}^{\alpha} p, \partial_{x}^{\alpha} g_{1}\right\rangle+\left\langle\partial_{x}^{\alpha} u, \partial_{x}^{\alpha} g_{2}\right\rangle \\
& =I_{1}+I_{2},
\end{aligned}
$$

where $I_{i}, i=1,2$ are the corresponding terms in the above equation which will be estimated as follows. Notice that under the condition (1.2) and (4.1), $\left(g_{1}, g_{2}\right)$ has the following equivalence properties:

$$
\begin{aligned}
g_{1} & \sim \partial_{i} p u_{i}+p \partial_{i} u_{i}+\Psi(u), \\
g_{2 j} & \sim u^{i} \partial_{i} u_{j}+p \partial_{i} \partial_{i} u_{j}+s \partial_{i} \partial_{i} u_{j}+p \partial_{j} \partial_{i} u_{i}+p \partial_{j} p+s \partial_{j} p+s \partial_{j} \partial_{i} u_{i} .
\end{aligned}
$$

Here and in the sequel proof, the repeated index denotes summation over the index.

Firstly, for $I_{1}$, it holds that

$$
\begin{aligned}
I_{1} \leq & C\left\{\left|\left\langle\partial_{x}^{\alpha} p, \partial_{x}^{\alpha}\left(\partial_{i} p u_{i}\right)\right\rangle\right|+\left|\left\langle\partial_{x}^{\alpha} p, \partial_{x}^{\alpha}\left(p \partial_{i} u_{i}\right)\right\rangle\right|+\left|\left\langle\partial_{x}^{\alpha} p, \partial_{x}^{\alpha} \Psi(u)\right\rangle\right|\right\} \\
\leq & C\left|\left\langle\partial_{x}^{\alpha} p, \partial_{x}^{\alpha} \partial_{i} p u_{i}\right\rangle\right|+C \sum_{|\beta| \leq|\alpha|-1} C_{\alpha}^{\beta}\left|\left\langle\partial_{x}^{\alpha} p, \partial_{x}^{\beta} \partial_{i} p \partial_{x}^{\alpha-\beta} u_{i}\right\rangle\right| \\
& +C \sum_{|\beta| \leq|\alpha|} C_{\alpha}^{\beta}\left|\left\langle\partial_{x}^{\alpha} p, \partial_{x}^{\beta} p \partial_{x}^{\alpha-\beta} \partial_{i} u_{i}\right\rangle\right|+C\left|\left\langle\partial_{x}^{\alpha} p, \partial_{x}^{\alpha} \Psi(u)\right\rangle\right| .
\end{aligned}
$$

For the first term on the right hand side of (4.18), from Lemma 3.2, one has

$$
\begin{aligned}
& \left|\left\langle\partial_{x}^{\alpha} p, \partial_{x}^{\alpha} \partial_{i} p u_{i}\right\rangle\right|=\frac{1}{2}\left|\left\langle\left(\partial_{x}^{\alpha} p\right)^{2}, \partial_{i} u_{i}\right\rangle\right| \\
& \leq C\left\|\partial_{i} u_{i}\right\|_{L^{\infty}}\left\|\partial_{x}^{\alpha} p\right\|^{2} \leq C\left\|\nabla^{2} u\right\|_{1}\left\|\partial_{x}^{\alpha} p\right\|^{2} \\
& \leq C \epsilon\left\|\partial_{x}^{\alpha} p\right\|^{2} .
\end{aligned}
$$

Furthermore, for the second term, it follows from the Hölder inequality and Lemma 
3.2 that

$$
\begin{aligned}
& \sum_{|\beta| \leq|\alpha|-1}\left|\left\langle\partial_{x}^{\alpha} p, \partial_{x}^{\beta} \partial_{i} p \partial_{x}^{\alpha-\beta} u_{i}\right\rangle\right| \\
= & \left\{\sum_{|\beta|=0}+\sum_{1 \leq|\beta| \leq|\alpha|-1}\right\}\left|\left\langle\partial_{x}^{\alpha} p, \partial_{x}^{\beta} \partial_{i} p \partial_{x}^{\alpha-\beta} u_{i}\right\rangle\right| \\
\leq & 2 \epsilon\left\|\partial_{x}^{\alpha} p\right\|^{2}+\frac{C}{\epsilon}\left\|\partial_{i} p \partial_{x}^{\alpha} u^{i}\right\|^{2}+\frac{C}{\epsilon} \sum_{1 \leq|\beta| \leq|\alpha|-1}\left\|\partial_{x}^{\alpha-\beta} u_{i} \partial_{x}^{\beta} \partial_{i} p\right\|^{2} \\
\leq & 2 \epsilon\left\|\partial_{x}^{\alpha} p\right\|^{2}+\frac{C}{\epsilon}\left\|\partial_{i} p\right\|_{L^{\infty}}^{2}\left\|\partial_{x}^{\alpha} u_{i}\right\|^{2}+\frac{C}{\epsilon} \sum_{1 \leq|\beta| \leq|\alpha|-1}\left\|\partial_{x}^{\alpha-\beta} u_{i}\right\|_{L^{\infty}}^{2}\left\|\partial_{x}^{\beta} \partial_{i} p\right\|^{2} \\
\leq & 2 \epsilon\left\|\partial_{x}^{\alpha} p\right\|^{2}+\frac{C}{\epsilon}\left\|\nabla \partial_{i} p\right\|_{1}^{2}\left\|\partial_{x}^{\alpha} u_{i}\right\|^{2}+\frac{C}{\epsilon} \sum_{1 \leq|\beta| \leq|\alpha|-1}^{\alpha-\beta}\left\|\nabla \partial_{x}^{\alpha-\beta} u_{i}\right\|_{1}^{2}\left\|\partial_{x}^{\beta} \partial_{i} p\right\|^{2} \\
\leq & C \epsilon \sum_{1 \leq|\alpha| \leq 3}\left\|\partial_{x}^{\alpha} p\right\|^{2}+C \epsilon \sum_{1 \leq|\alpha| \leq 4}\left\|\partial_{x}^{\alpha} u\right\|^{2} .
\end{aligned}
$$

The third and fourth terms on the right hand side of (4.18) can be estimated similarly. Thus,

$$
I_{1} \leq C \epsilon \sum_{1 \leq|\alpha| \leq 3}\left\|\partial_{x}^{\alpha} p\right\|^{2}+C \epsilon \sum_{1 \leq|\alpha| \leq 4}\left\|\partial_{x}^{\alpha} u\right\|^{2} .
$$

Moreover, $I_{2}$ can be estimated similarly as $I_{1}$. Then

$$
I_{2} \leq C \epsilon \sum_{1 \leq|\alpha| \leq 3}\left\|\partial_{x}^{\alpha} p\right\|^{2}+C \epsilon \sum_{1 \leq|\alpha| \leq 4}\left\|\partial_{x}^{\alpha} u\right\|^{2} .
$$

Hence (4.17) together with (4.19) and (4.20) yields

$$
\begin{aligned}
& \frac{d}{d t} \sum_{1 \leq|\alpha| \leq 3}\left\|\partial_{x}^{\alpha}(p, u)\right\|^{2}+\sum_{1 \leq|\alpha| \leq 3}\left\|\nabla \partial_{x}^{\alpha} u\right\|^{2} \\
\leq & C \epsilon \sum_{1 \leq|\alpha| \leq 3}\left\|\partial_{x}^{\alpha} p\right\|^{2}+C \epsilon \sum_{1 \leq|\alpha| \leq 4}\left\|\partial_{x}^{\alpha} u\right\|^{2} .
\end{aligned}
$$

Next, for the estimate on $\left\|\nabla \partial_{x}^{\alpha} p\right\|^{2}$ for $1 \leq|\alpha| \leq 2$, as in [5], we have

$$
\begin{aligned}
& \frac{\lambda_{1}}{2} \sum_{1 \leq|\alpha| \leq 2}\left\|\nabla \partial_{x}^{\alpha} p\right\|^{2}+\frac{d}{d t} \sum_{1 \leq|\alpha| \leq 2}\left\langle\partial_{x}^{\alpha} u, \nabla \partial_{x}^{\alpha} p\right\rangle \\
\leq & C \sum_{1 \leq|\alpha| \leq 2}\left\|\partial_{x}^{\alpha} \nabla u\right\|_{1}^{2}+C \epsilon \sum_{1 \leq|\alpha| \leq 3}\left\|\partial_{x}^{\alpha} p\right\|^{2}+C \epsilon \sum_{1 \leq|\alpha| \leq 4}\left\|\partial_{x}^{\alpha} u\right\|^{2} .
\end{aligned}
$$

Since $\epsilon>0$ is sufficiently small, multiplying (4.21) by $D_{2}$ suitably large and adding it to (4.22) give (4.16), and this completes the proof of the lemma.

Finally we consider the energy estimates on the entropy $s$.

Lemma 4.3. It holds that

$$
\frac{d}{d t}\|s(t)\|_{3}^{2} \leq C\left\|\nabla^{2} u(t)\right\|_{1}\|s(t)\|_{3}^{2}+C \epsilon\|\nabla u(t)\|_{3}^{2},
$$

for any $0 \leq t \leq T$. 
Proof. For each multi-index $\alpha$ with $0 \leq|\alpha| \leq 3$, by applying $\partial_{x}^{\alpha}$ to $(2.1)_{3}$, multiplying it by $\partial_{x}^{\alpha} s$, integrating it over $\mathbb{R}^{3}$ and then summing them up, we have

$$
\begin{aligned}
\frac{1}{2} \frac{d}{d t}\|s\|_{3}^{2} & =-\lambda \sum_{0 \leq|\alpha| \leq 3}\left\langle\partial_{x}^{\alpha}(u \cdot \nabla s), \partial_{x}^{\alpha} s\right\rangle+\sum_{0 \leq|\alpha| \leq 3}\left\langle\partial_{x}^{\alpha} g_{3}, \partial_{x}^{\alpha} s\right\rangle \\
& =J_{1}+J_{2}
\end{aligned}
$$

Here $J_{1}$ and $J_{2}$, which are the corresponding terms on the right hand side, are estimated as follows. For $J_{1}$, one has

$$
J_{1}=-\lambda \sum_{0 \leq|\alpha| \leq 3}\left\langle u \cdot \nabla \partial_{x}^{\alpha} s, \partial_{x}^{\alpha} s\right\rangle-\lambda \sum_{\substack{0 \leq \beta \leq|\alpha|-1 \\ 1 \leq|\alpha| \leq 3}}\left\langle\partial_{x}^{\alpha-\beta} u \cdot \nabla \partial_{x}^{\beta} s, \partial_{x}^{\alpha} s\right\rangle,
$$

where for each $\alpha$ with $0 \leq \alpha \leq 3$, it holds that

$$
\left\langle u, \nabla \partial_{x}^{\alpha} s, \partial_{x}^{\alpha} s\right\rangle=-\frac{1}{2}\left\langle\nabla \cdot u,\left(\partial_{x}^{\alpha} s\right)^{2}\right\rangle \leq\|\nabla \cdot u\|_{L^{\infty}}\left\|\partial_{x}^{\alpha} s\right\|^{2} \leq\left\|\nabla^{2} u\right\|_{1}\left\|\partial_{x}^{\alpha} s\right\|^{2},
$$

and for each $\alpha, \beta$ with $1 \leq|\alpha| \leq 3,0 \leq \beta \leq|\alpha|-1$, we have

$$
\begin{aligned}
\left\langle\partial_{x}^{\alpha-\beta} u \cdot \nabla \partial_{x}^{\beta} s, \partial_{x}^{\alpha} s\right\rangle \leq \begin{cases}\left\|\partial_{x}^{\alpha} u\right\|_{L^{\infty}}\|\nabla s\|\left\|\partial_{x}^{\alpha} s\right\|, & \text { if }|\beta|=0,|\alpha|=1 \\
\left\|\partial_{x}^{\alpha} u\right\|\|\nabla s\|_{L^{\infty}}\left\|\partial_{x}^{\alpha} s\right\|, & \text { if }|\beta|=0,2 \leq|\alpha| \leq 3 \\
\left\|\partial_{x}^{\alpha-\beta} u\right\|_{L^{6}}\left\|\nabla \partial_{x}^{\beta} s\right\|_{L^{3}}\left\|\partial_{x}^{\alpha} s\right\|, & \text { if }|\beta|=1,2 \leq|\alpha| \leq 3 \\
\left\|\partial_{x}^{\alpha-\beta} u\right\|_{L^{\infty}}\left\|\nabla \partial_{x}^{\beta} s\right\|\left\|\partial_{x}^{\alpha} s\right\|, & \text { if }|\beta|=2,|\alpha|=3\end{cases} \\
\leq\left\|\nabla^{2} u\right\|_{1}\|\nabla s\|_{2}^{2} .
\end{aligned}
$$

Hence, it holds that

$$
J_{1} \leq C\left\|\nabla^{2} u\right\|_{1}\|s\|_{3}^{2} .
$$

For $J_{2}$, since $\Psi(u)$ is quadratically nonlinear, using (4.1) we have

$$
\begin{aligned}
J_{2} \leq & \sum_{0 \leq|\alpha| \leq 3}\left\|\partial_{x}^{\alpha} g_{3}\right\|\left\|\partial_{x}^{\alpha} s\right\| \leq \epsilon \sum_{0 \leq|\alpha| \leq 3}\left\|\partial_{x}^{\alpha}\left(\frac{\Psi(\lambda u)}{p+p_{\infty}}\right)\right\| \\
\leq & \epsilon \sum_{\substack{\beta \leq \alpha \\
0 \leq|\alpha| \leq 3}} C_{\alpha}^{\beta}\left\|\partial_{x}^{\alpha-\beta}\left(\frac{1}{p+p_{\infty}}\right) \partial_{x}^{\beta} \Psi(\lambda u)\right\| \\
\leq & C \epsilon\left(\sum_{\substack{\beta \leq \alpha \\
2 \leq|\beta| \leq|\alpha| \leq 3}}\left\|\partial_{x}^{\alpha-\beta}\left(\frac{1}{p+p_{\infty}}\right)\right\|_{L^{\infty}}\left\|\partial_{x}^{\beta} \Psi(\lambda u)\right\|\right. \\
& \left.+\sum_{\substack{|\beta| \leq 1, \beta \leq \alpha \\
|\beta| \leq|\alpha| \leq 3}}\left\|\partial_{x}^{\alpha-\beta}\left(\frac{1}{p+p_{\infty}}\right)\right\|\left\|\partial_{x}^{\beta} \Psi(\lambda u)\right\|_{L^{\infty}}\right) \\
\leq & C \epsilon\|\nabla u\|_{3}^{2} .
\end{aligned}
$$

Combining (4.24), (4.25) and (4.26) yields (4.23), and this completes the proof of the lemma. 
Remark 4.1. Up to now, one only can close a priori assumption about $(p, u)$ from Lemmas 4.1 and 4.2. Since there is not any dissipation rate on the entropy $s$ in the inequality (4.23), a priori assumption on s can not be closed for this moment. In order to close a priori assumption on s, we have to study a priori decay-in-time estimates on $u$. In fact, from Lemma 4.3 together with Lemmas 4.1 and 4.2, we can obtain the uniform bound of $s$ if $\left\|\nabla^{2} u\right\|_{1}$ decays in time at some rate fast enough, which is given in the next section.

\section{Global existence and convergence rate}

In order to prove Proposition 2.2, in this section we continue to consider a priori decay-in-time estimates on $(p, u)$. This will be based on Lemma 3.1 about the decay estimates on the semigroup $e^{-t \mathbb{A}}$. Precisely, we have the following lemma.

Lemma 5.1. $(p, u)$ satisfies the inequality

$$
\|\nabla(p, u)(t)\| \leq C K_{0}(1+t)^{-\frac{5}{4}}+C \epsilon \int_{0}^{t}(1+t-\tau)^{-\frac{5}{4}}\|\nabla(p, u)(\tau)\|_{2} d \tau,
$$

for any $0 \leq t \leq T$, where $K_{0}=\left\|\left(p_{0}, u_{0}\right)\right\|_{L^{1} \cap H^{3}}$ as in (2.4) is finite by the assumption of Proposition 2.2.

Proof. From the Duhamel's principle, it holds that

$$
(p, u)(t)=e^{-t \mathbb{A}}\left(p_{0}, u_{0}\right)+\int_{0}^{t} e^{-(t-\tau) \mathbb{A}}\left(g_{1}, g_{2}\right)(\tau) d \tau
$$

Thus from Lemma 3.1, we have

$$
\begin{aligned}
\|\nabla(p, u)(t)\| \leq & C K_{0}(1+t)^{-\sigma(1,2 ; 1)} \\
& +C \int_{0}^{t}(1+t-\tau)^{-\sigma(1,2 ; 1)}\left\|\left(g_{1}, g_{2}\right)(\tau)\right\|_{L^{1} \cap H^{1}} d \tau
\end{aligned}
$$

where $\sigma(1,2 ; 1)=\frac{5}{4}$ by $(3.2)$. The nonlinear source terms can be estimated as follows:

$$
\begin{aligned}
& \left\|\left(g_{1}, g_{2}\right)(t)\right\|_{L^{1}} \leq C\|(p, u, s)(t)\|_{1}\|\nabla(p, u)(t)\|_{1} \leq C \epsilon\|\nabla(p, u)(t)\|_{1}, \\
& \left\|\left(g_{1}, g_{2}\right)(t)\right\|_{1} \leq C\|(p, u, s)(t)\|_{W^{1, \infty}}\|\nabla(p, u)(t)\|_{2} \leq C \epsilon\|\nabla(p, u)(t)\|_{2} .
\end{aligned}
$$

Putting these estimates into (5.2) yields (5.1), and this completes the proof of the lemma.

Now we are in a position to prove Proposition 2.2. 
Proof of Proposition 2.2 We will do it by three steps.

Step 1. Since $\epsilon>0$ is sufficiently small, from Lemma 4.1 and Lemma 4.2, we can choose a constant $D_{3}>0$ suitably large such that

$$
\frac{d}{d t}\left(D_{3}\|(p, u)(t)\|_{3}^{2}+\sum_{|\alpha| \leq 2}\left\langle\nabla \partial_{x}^{\alpha} p, \partial_{x}^{\alpha} u\right\rangle(t)\right)+C\left(\|\nabla p(t)\|_{2}^{2}+\|\nabla u(t)\|_{3}^{2}\right) \leq 0,
$$

for any $0 \leq t \leq T$, which implies

$$
\|(p, u)(t)\|_{3}^{2}+\int_{0}^{t}\left(\|\nabla p(\tau)\|_{2}^{2}+\|\nabla u(\tau)\|_{3}^{2}\right) d \tau \leq C\left\|\left(p_{0}, u_{0}\right)\right\|_{3}^{2},
$$

since

$$
D_{3}\|(p, u)(t)\|_{3}^{2}+\sum_{|\alpha| \leq 2}\left\langle\nabla \partial_{x}^{\alpha} p, \partial_{x}^{\alpha} u\right\rangle(t) \sim\|(p, u)(t)\|_{3}^{2} .
$$

Then (5.5) gives (2.2).

Step 2. Define the temporal energy functional

$$
H(t)=D_{2}\|\nabla(p, u)(t)\|_{2}^{2}+\sum_{1 \leq|\alpha| \leq 2}\left\langle\partial_{x}^{\alpha} u, \nabla \partial_{x}^{\alpha} p\right\rangle(t),
$$

for any $0 \leq t \leq T$, where it is noticed that $H(t)$ is equivalent to $\|\nabla(p, u)(t)\|_{2}^{2}$ since $D_{2}$ can be large enough, i.e. there exists a constant $C>1$, such that

$$
\frac{1}{C}\|\nabla(p, u)(t)\|_{2}^{2} \leq H(t) \leq C\|\nabla(p, u)(t)\|_{2}^{2} .
$$

From Lemma 4.2, we have

$$
\frac{d H(t)}{d t}+C\left\|\nabla^{2}(p, u)(t)\right\|_{1}^{2} \leq C \epsilon\|\nabla(p, u)(t)\|^{2} .
$$

Adding $\|\nabla(p, u)(t)\|^{2}$ to both sides of the above inequality gives

$$
\frac{d H(t)}{d t}+D_{4} H(t) \leq C\|\nabla(p, u)(t)\|^{2},
$$

where $D_{4}$ is a positive constant independent of $\epsilon$. As in [4], define

$$
M(t)=\sup _{0 \leq \tau \leq t}(1+\tau)^{\frac{5}{2}} H(\tau)
$$

Notice that

$$
\|\nabla(p, u)(\tau)\|_{2} \leq C \sqrt{H(\tau)} \leq C(1+\tau)^{-\frac{5}{4}} \sqrt{M(t)}, \quad 0 \leq \tau \leq t \leq T .
$$

Then it follows from (5.1) that

$$
\begin{aligned}
\|\nabla(p, u)(t)\| & \leq C K_{0}(1+t)^{-\frac{5}{4}}+C \epsilon \int_{0}^{t}(1+t-\tau)^{-\frac{5}{4}}(1+\tau)^{-\frac{5}{4}} d \tau \sqrt{M(t)} \\
& \leq C(1+t)^{-\frac{5}{4}}\left(K_{0}+\epsilon \sqrt{M(t)}\right)
\end{aligned}
$$


where we have used Lemma 3.3. Hence, by the Gronwall's inequality, (5.6) and (5.9) lead to

$$
\begin{aligned}
H(t) & \leq H(0) e^{-D_{4} t}+C \int_{0}^{t} e^{-D_{4}(t-\tau)}\|\nabla(p, u)(\tau)\|^{2} d \tau \\
& \leq H(0) e^{-D_{4} t}+C \int_{0}^{t} e^{-D_{4}(t-\tau)}(1+\tau)^{-\frac{5}{2}} d \tau\left(K_{0}^{2}+\epsilon^{2} M(t)\right) \\
& \leq C(1+t)^{-\frac{5}{2}}\left(H(0)+K_{0}^{2}+\epsilon^{2} M(t)\right) .
\end{aligned}
$$

Since $M(t)$ is non-decreasing, we have from (5.10) that

$$
M(t) \leq C\left(H(0)+K_{0}^{2}+\epsilon^{2} M(t)\right)
$$

for any $0 \leq t \leq T$, which implies that

$$
M(t) \leq C\left(H(0)+K_{0}^{2}\right) \leq C K_{0}^{2},
$$

since $\epsilon>0$ is small enough. Thus (5.8) gives

$$
\|\nabla(p, u)(t)\|_{2} \leq C K_{0}(1+t)^{-\frac{5}{4}}, \quad 0 \leq t \leq T,
$$

which also implies from Lemma 3.2 that

$$
\|(p, u)(t)\|_{L^{\infty}} \leq C\|\nabla(p, u)(t)\|_{1} \leq C K_{0}(1+t)^{-\frac{5}{4}}, \quad 0 \leq t \leq T .
$$

Hence (2.6) and (2.7) are proved.

Next, using Lemma 3.1 and (5.3), it follows from the Duhamel's principle that

$$
\begin{aligned}
\|(p, u)(t)\| & \leq C K_{0}(1+t)^{-\frac{3}{4}}+C \int_{0}^{t}(1+t-\tau)^{-\frac{3}{4}}\left\|\left(g_{1}, g_{2}\right)(\tau)\right\|_{L^{1} \cap L^{2}} d \tau \\
& \leq C K_{0}(1+t)^{-\frac{3}{4}}+C \epsilon \int_{0}^{t}(1+t-\tau)^{-\frac{3}{4}}\|\nabla(p, u)(\tau)\|_{2} d s \\
& \leq C K_{0}(1+t)^{-\frac{3}{4}}+C \epsilon K_{0} \int_{0}^{t}(1+t-\tau)^{-\frac{3}{4}}(1+\tau)^{-\frac{5}{4}} d \tau \\
& \leq C K_{0}(1+t)^{-\frac{3}{4}}
\end{aligned}
$$

for any $0 \leq t \leq T$. On the other hand, using Lemma 3.2 we have

$$
\|(p, u)(t)\|_{L^{6}} \leq C\|\nabla(p, u)(t)\| \leq C K_{0}(1+t)^{-\frac{5}{4}} .
$$

Hence, by the interpolation, it holds that for any $2 \leq q \leq 6$,

$$
\|(p, u)(t)\|_{L^{q}} \leq\|(p, u)(t)\|_{L^{6}}^{\theta}\|(p, u)(t)\|^{1-\theta} \leq C K_{0}(1+t)^{-\frac{3}{2}\left(1-\frac{1}{q}\right)},
$$

for any $0 \leq t \leq T$, where $\theta=\frac{3(q-2)}{2 q}$. Thus (2.5) is proved.

Step 3. Since $\epsilon>0$ is small enough, from Lemma 4.3 and (5.4), we have

$$
\frac{d}{d t}\left(D_{3}\|(p, u)(t)\|_{3}^{2}+\|s(t)\|_{3}^{2}+\sum_{|\alpha| \leq 2}\left\langle\nabla \partial_{x}^{\alpha} p, \partial_{x}^{\alpha} u\right\rangle(t)\right) \leq C\left\|\nabla^{2} u\right\|_{1}\|s\|_{3}^{2} .
$$


Let

$$
y(t)=D_{3}\|(p, u)(t)\|_{3}^{2}+\|s(t)\|_{3}^{2}+\sum_{|\alpha| \leq 2}\left\langle\nabla \partial_{x}^{\alpha} p, \partial_{x}^{\alpha} u\right\rangle(t) .
$$

Since $D_{3}>0$ is large enough, then it holds that

$$
y(t) \sim\|(p, u, s)(t)\|_{3}^{2} .
$$

Thus, from (5.11), we have

$$
\frac{d}{d t} y(t) \leq C\left\|\nabla^{2} u\right\|_{1} y(t),
$$

which by the Gronwall's inequality gives

$$
\begin{aligned}
y(t) & \leq y(0) \exp \left\{C \int_{0}^{t}\left\|\nabla^{2} u(\tau)\right\| d \tau\right\} \\
& \leq y(0) \exp \left\{C K_{0} \int_{0}^{t}(1+\tau)^{-\frac{5}{4}} d \tau\right\} \\
& \leq y(0) \exp \left\{C K_{0}\right\},
\end{aligned}
$$

i.e., for any $0 \leq t \leq T$,

$$
\|(p, u, s)(t)\|_{3}^{2} \leq C\left\|\left(p_{0}, u_{0}, s_{0}\right)\right\|_{3}^{2} \exp \left\{C K_{0}\right\} .
$$

Hence (2.3) holds. For (2.8), using the above estimates and (2.1), we have

$$
\begin{aligned}
\left\|\partial_{t}(p, u, s)(t)\right\| \leq & \left\|\lambda_{1} \nabla \cdot u(t)\right\|+\left\|g_{1}(t)\right\|+\left\|\lambda_{1} \nabla p(t)-\mu_{1} \Delta u(t)-\mu_{2} \nabla \nabla \cdot u(t)\right\| \\
& +\left\|g_{2}(t)\right\|+\|\lambda(u \cdot \nabla s)(t)\|+\left\|g_{3}(t)\right\| \\
\leq & C\left(\|\nabla u(t)\|_{1}+\|\nabla p(t)\|+\|\nabla p(t)\|_{1}\|\nabla u(t)\|+\|\nabla u(t)\|^{2}\right. \\
& +\|\nabla u(t)\|_{1}\|\nabla(p, u, s)(t)\|+\|\nabla(p, s)(t)\|_{1}\left(\|\nabla p(t)\|+\left\|\nabla^{2} u(t)\right\|\right) \\
\leq & C K_{0}(1+t)^{-\frac{5}{4}},
\end{aligned}
$$

for any $0 \leq t \leq T$. Thus, (2.8) is proved and this completes the proof of Proposition 2.2 .

\section{References}

[1] R. Adams, Sobolev spaces. New York, Academic Press, 1985.

[2] K. Deckelnick, Decay estimates for the compressible Navier-Stokes equations in unbounded domains, Math. Z. 209 (1992), 115-130.

[3] K. Deckelnick, $L^{2}$-decay for the compressible Navier-Stokes equations in unbounded domains, Comm. Partial Differential Equations 18 (1993), 1445-1476. 
[4] R.-J. Duan, H.-X. Liu, S. Ukai and T. Yang, Optimal $L^{p}-L^{q}$ convergence rate for the compressible Navier-Stokes equations with potential force, J. Differential Equations 238 (2007), 220-233.

[5] R.-J. Duan, S. Ukai, T. Yang and H.-J. Zhao, Optimal convergence rate for the compressible Navier-Stokes equations with potential force, Math. Mod. Meth. Appl. Sci. 17 (2007), 737-758.

[6] D. Hoff and K. Zumbrun, Multidimensional diffusion waves for the Navier-Stokes equations of compressible flow, Indiana Univ. Math. J. 44 (1995), 604-676.

[7] D. Hoff and K. Zumbrun, Pointwise decay estimates for multidimensional Navier-Stokes diffusion waves, Z. angew. Math. Phys. 48 (1997), 597-614.

[8] S. Kawashima, Systems of a hyperbolic-parabolic composite type, with applications to the equations of magnetohydrodynamics, thesis, Kyoto University, 1983.

[9] S. Kawashima and M. Okada, Smooth global solutions for the one-dimensional equations in magneto-hydrodynamics, Proc. Japan Acad. Ser. A Math. Sci. 58 (1982), 384387 .

[10] T. Kobayashi, Some estimates of solutions for the equations of motion of compressible viscous fluid in an exterior domain in $\mathbb{R}^{3}$, J. Differential equations 184 (2002), 587-619.

[11] T. Kobayashi and Y. Shibata, Decay estimates of solutions for the equations of motion of compressible viscous and heat-conductive gases in an exterior domain in $\mathbf{R}^{3}$, Commun. Math. Phys. 200 (1999), 621-659.

[12] T.-P. Liu and W.-K. Wang, The pointwise estimates of diffusion waves for the NavierStokes equations in odd multi-dimensions, Commun. Math. Phys. 196 (1998), 145-173.

[13] T.-P. Liu and Y. Zeng, Compressible Navier-Stokes equations with zero heat conductivity, J. Diff. Eqns. 153 (1999), 225-291.

[14] T.-P. Liu and Y. Zeng, Large time behavior of solutions for general quasilinear hyperbolic- parabolic sustems of conservation laws, Mem. Amer. Math. Soc. 125 (1997), 599 .

[15] A. Matsumura and T. Nishida, The initial value problem for the equations of motion of compressible viscous and heat-conductive fluids, Proc. Japan Acad. Ser. A 55 (1979), $337-342$.

[16] A. Matsumura and T. Nishida, The initial value problems for the equations of motion of viscous and heat-conductive gases, J. Math. Kyoto Univ. 20 (1980), 67-104.

[17] G. Ponce, Global existence of small solutions to a class of nonlinear evolution equations, Nonlinear Anal. 9 (1985), 339-418.

[18] S. Ukai, T. Yang and H.-J. Zhao, Convergence rate for the compressible Navier-Stokes equations with external force, J. Hyperbolic Differ. Equ. 3 (2006), 561-574.

[19] Y. Zeng, Gas Dynamics in thermal Nonequilibrium and general Hyperbolic Systems with Relaxation, Arch. Rational Mech. Anal. 150 (1999), 225-297.

[20] Y. Zeng, $L^{1}$ asymptotic behavior of compressible, isentropic, viscous 1-D flow, Comm. Pure Appl. Math. 47 (1994), 1053-1082. 